\title{
Ovarian preservation with lateral ovarian transposition in operable Ca cervix: experience at a tertiary care center
}

\author{
${\text { Tony } \text { Jose }^{1 *} \text {, Amarinder Singh }}^{2}$, Pooja Sinha ${ }^{3}$, Sumit Bidari $^{1}$
}

\begin{abstract}
${ }^{1}$ Department of Obstetrics and Gynecology, Armed Forces Medical College, Pune, Maharashtra, India ${ }^{2}$ Department of Obstetrics and Gynecology, Military Hospital, Amritsar, Punjab, India

${ }^{3}$ Department of Obstetrics and Gynecology, Army College of Medical Sciences, New Delhi, India
\end{abstract}

Received: 11 May 2017

Accepted: 16 May 2017

\author{
*Correspondence: \\ Dr. Tony Jose, \\ E-mail: oncotango@gmail.com
}

Copyright: () the author(s), publisher and licensee Medip Academy. This is an open-access article distributed under the terms of the Creative Commons Attribution Non-Commercial License, which permits unrestricted non-commercial use, distribution, and reproduction in any medium, provided the original work is properly cited.

\begin{abstract}
Background: The benefits of ovarian preservation during radical surgery for ca cervix in premenopausal patients far outweigh the risk of ovarian disease later. However, adjuvant pelvic radiotherapy damages retained ovaries. Mobilization and transposition of these ovaries during surgery outside the pelvis prevents or reduces radiation damage to these ovaries. A prospective observational study was conducted to objectively study the effect of ovarian preservation with Lateral Ovarian Transposition (LOT) on ovarian function on operated patients of Ca cervix.

Methods: All pre-menopausal patients of Ca cervix <45 years, planned for surgery underwent FSH levels to assess ovarian function and were screened for ovarian disease. Eligible patients who consented underwent ovarian retention (OR) with LOT during surgery. Adjuvant treatments as indicated was administered and all patients were followed up at 3,6 and 12 months after surgery for subjective (symptoms) and objective (FSH) evidence of ovarian failure.

Results: LOT could be successfully performed in all patients. Though the mean FSH values did show a rise after surgery, this was more pronounced in patients receiving radiotherapy (RT). Ovarian function continued normally in $63 \%$ of all patients with $72 \%$ retaining ovarian function when RT was not administered. Hot flashes (HF) and Vaginal dryness(VD) were the most common symptoms but its onset was gradual and symptoms milder compared to surgical menopause.

Conclusions: LOT is an oncologicaly safe, technically feasible and reasonably effective procedure for preserving ovarian function during surgical treatment and even after adjuvant RT in Ca Cervix and should be offered to eligible premenopausal patients.
\end{abstract}

Keywords: Carcinoma cervix, Follicle stimulating hormone, Hot flashes, Lateral Ovarian transposition, Ovarian retention

\section{INTRODUCTION}

About $40 \%$ of cervical malignancies occur in premenopausal age groups. Conventional treatment of $\mathrm{Ca}$ cervix causes premature menopause in young patients due to oophorectomy done at surgery or damage to ovaries during radiotherapy (RT). McCall et al first studied ovarian retention at the time of radical hysterectomy and found that retained ovaries continued to function for many years after surgery. ${ }^{1}$ Ovarian preservation has been a major advantage of surgical treatment of Ca cervix ever since Webb et al and Landoni et al established the oncological safety of ovarian retention (OR). ${ }^{2,3}$ Gubbala et al in their meta-analysis has brought out many additional benefits of OR. ${ }^{4}$ Adjuvant RT damages the ovaries at a cumulative dose of 6-20 G thus eliminating the advantage of OR. ${ }^{5}$ Surgical techniques were hence devised to transpose the ovaries 
out of the radiation field in the pelvis and methods of abdominal shielding during RT were developed to reduce the damage to the ovaries. ${ }^{6,7}$ This prospective observational study was done to objectively evaluate the ovarian function after lateral ovarian transposition (LOT) during radical hysterectomy $(\mathrm{RH})$ in patients with operable ca cervix with and without RT over a period of 12 months.

\section{METHODS}

All pre-menopausal patients $<45$ years with operable Squamous and Adeno-squamous Ca Cervix up to stage IIA $^{1}$ reporting for treatment for a 3-year period from Jan 2013 to Jan 2016 were included in the study. Adenocarcinomas, ovarian pathologies, those with family histories of breast, ovarian or uterine malignancies and patients with high $\mathrm{FSH} / \mathrm{LH}$ levels (>20 IU/ml) were excluded from the study. The pre-operative work up included, TVS for evaluation of ovaries (CECT in case of bulky cervical disease), Ca 125 levels and Day 2 FSH levels. All eligible patients were counselled for OR with LOT and a written informed consent obtained. During surgery, a midline incision was used, both ovaries evaluated and LOT was performed according to the method of Belinson et al. ${ }^{8}$ Ovaries were detached from the uterus and tubes carefully preserving the vascularity and they were mobilized on the IP ligament and packed into the abdominal cavity. Suspicious lymph nodes (pelvic and/or para aortic) if any were sampled and sent for frozen section. Piver's type III Radical hysterectomy and bilateral pelvic lymphadenectomy was performed. ${ }^{9}$ $\mathrm{RH}$ was abandoned in case of nodal metastasis. The mobilized ovaries were once again evaluated for the intactness of vascularity and thereafter were fixed in the lumbar region near the lower pole of the kidney. The location of the ovaries was marked with two intersecting titanium clips so as to be visible on subsequent X-rays.

Out of 206 patients with Ca Cervix, 64 were considered operable and planned for Type III RH of which 28 patients were premenopausal and considered for LOT. However, 3 patients were $>45$ years and 2 patients had adenocarcinoma on biopsy and hence were excluded. The remaining 23 patients were counselled for OR, out of which two refused consent. 21 patients underwent laparotomy and 19 patients finally underwent OR with LOT which included 2 patients in whom RH was abandoned one due to bladder infiltration and the other found to have nodal metastasis on frozen section but LOT was performed. Two patients had to be subjected to BSO, one with bilateral dermoid cysts and the other for severe endometriosis. Two patients underwent unilateral LOT as one had a unilateral dermoid and the second patient was found to have only one ovary. Adjuvant postoperative treatment was administered according to the histologic results (occult parametrial disease, nodal metastasis, positive vaginal cut margins, lympho-vascular space invasion (LVSI), deep stromal invasion and tumor size $>2 \mathrm{~cm}$ ) in the form of External beam radiotherapy
(EBRT) vaginal Brachytherapy (BT) with or without weekly Cisplatin and was administered with additional abdominal shielding during EBRT. 3 patients received extended field RT (EFRT) where shielding of abdomen was not possible. Patients were evaluated after 3 months for evidence of acute estrogen withdrawal in the form of menopausal symptoms (hot flashes, vaginal dryness) and FSH levels. FSH levels above $20 \mathrm{mIU} / \mathrm{ml}$ were considered abnormal. This evaluation was repeated at each follow up visits at, 3 months, 6 months, and 1 year after surgery.

\section{RESULTS}

The mean age of the patients in this study was 41.52 years (Range 38-45 years) with a median age of 41.00 . The mean BMI of patients in this study was $23.28 \mathrm{Kg} / \mathrm{m}^{2}$ $\left(18.4-34.4 \mathrm{Kg} / \mathrm{m}^{2}\right)$. The mean gravidity was 2.6 and mean parity was 2.1 . The FIGO stages included 1 case of Stage IA $^{1}, 2$ of Stage I A ${ }^{2}, 9$ of stage IB $^{1}, 6$ cases of $\mathrm{I} \mathrm{B}^{2}$ and 3 of stage IIA ${ }^{1}$. The tumor histologies included squamous cell carcinomas in 16 and adenosquamous carcinoma in 3. (Table 1) The mean pre-operative FSH levels in the study was $7.62 \mathrm{mIU} / \mathrm{ml}$ (3.6-16.10) and the mean levels 3,6 , and 12 months after surgery were 13.66, 20.54 and $22.453 \mathrm{mIU} / \mathrm{ml}$. There were no immediate post-operative complications related to LOT. Out of the 19 patients who underwent LOT (unilateral in 2 patients), 11 patients did not receive RT while 8 patients underwent adjuvant RT 5 patients received EBRT and 3 patients received EFRT.

Table 1: Patient variables and outcomes.

\begin{tabular}{|llll|}
\hline Variable & $\begin{array}{l}\text { No RT } \\
\text { Group }\end{array}$ & $\begin{array}{l}\text { RT } \\
\text { Group }\end{array}$ & Total \\
\hline Mean age (years) & 41.81 & 41.65 & 41.52 \\
\hline Age & & & \\
\hline$<40$ Years & 2 & 0 & 02 \\
\hline$>40$ Years & 09 & 08 & 17 \\
\hline Tumor stage (FIGO) & 01 & 0 & 01 \\
\hline IA1 & 02 & 0 & 02 \\
\hline 1A2 & 05 & 04 & 09 \\
\hline IB1 & 02 & 04 & 06 \\
\hline IB2 & 03 & 0 & 03 \\
\hline IIA1 & & & \\
\hline Histology & 09 & 08 & 17 \\
\hline Squamous cell Ca & 09 & 0 & 02 \\
\hline Adenosquamous Ca & 02 & & \\
\hline Nodal status & & 03 & 14 \\
\hline N- & 11 & 03 & 03 \\
\hline N+(Pelvic) & 0 & 02 & 02 \\
\hline N+(Para Aortic) & 0 & 08 & 19 \\
\hline Total & 11 & 04 & $12(63 \%)$ \\
\hline Functional ovaries & $08(71 \%)$ & $(50 \%)$ & \\
\hline @ 12 m & & & \\
\hline
\end{tabular}

Though the FSH levels showed a continuously rising trend even after OR, there was a distinct difference in the groups that were exposed to RT. In the No RT group the 
mean baseline FSH level was $7.14 \mathrm{mIU} / \mathrm{ml}$ and post op levels at 3, 6 and 12 months were 9.70, 12.78 and 13.66 $\mathrm{mIU} / \mathrm{ml}$ respectively. Thus, the ovarian function continued and the rise in FSH levels though apparent was not statistically significant.

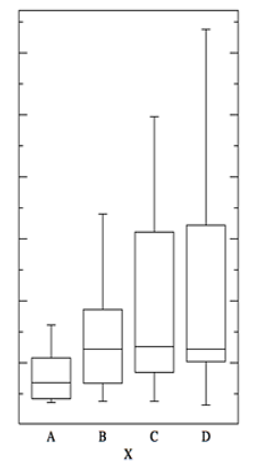

All Patients

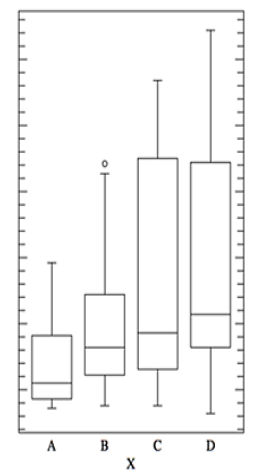

No RT Group

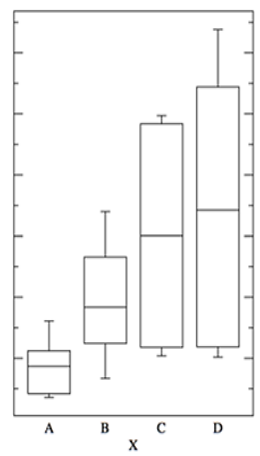

RT Group
Figure 1: Mean FSH Values.

However, in the group that underwent EBRT, though the mean pre-operative values were comparable (mean FSH$8.48 \mathrm{mIU} / \mathrm{ml}$ ), the post-operative values at, 3,6 and 12 months showed a steady rise, (19.0, 30.20 and $34.75 \mathrm{mIU} / \mathrm{ml}$ respectively). Though this suggested that ovarian function declined steadily despite OR with LOT, further analysis shows that this decline was much more pronounced in patients who underwent EFRT for para aortic nodal metastasis. 3 out of the 8 patients who underwent RT received EFRT and all three of them had very high FSH levels right from the $3^{\text {rd }}$ month. This was due to inclusion of the transplanted ovaries in the field of RT and inability to use shielding during EFRT. Among the 5 patients who underwent EBRT only, ovaries remained functional at 3 month follow up in all and 3 patients had continuing ovarian function at 12 months follow up $(60 \%)$.

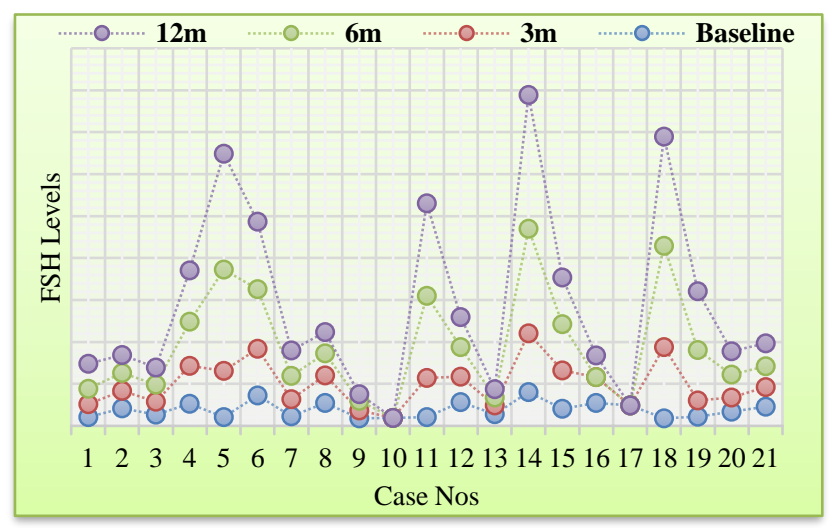

Figure 3: FSH Follow up.

On analysis of the symptoms of estrogen withdrawal, it was found that after 3 months of surgery, 5 patients had HF (5/19), two of whom underwent EFRT. Two patients from the RT group continued to have HF by 6 months $(5 / 19)$ but none had HF at one year follow up. One patient from each group complained of vaginal dryness (VD) (2/19) after 3 months and the numbers increased to 7 (3 from No RT and 4 from RT group) after brachytherapy, 6 of whom complained of the same after 12 months too. However, it was observed that the vasomotor symptoms were milder, and did not require estrogen for symptom relief in most patients except in 2 patients who had undergone EFRT. Vasomotor symptoms had subsided completely in all patients by 12 months.

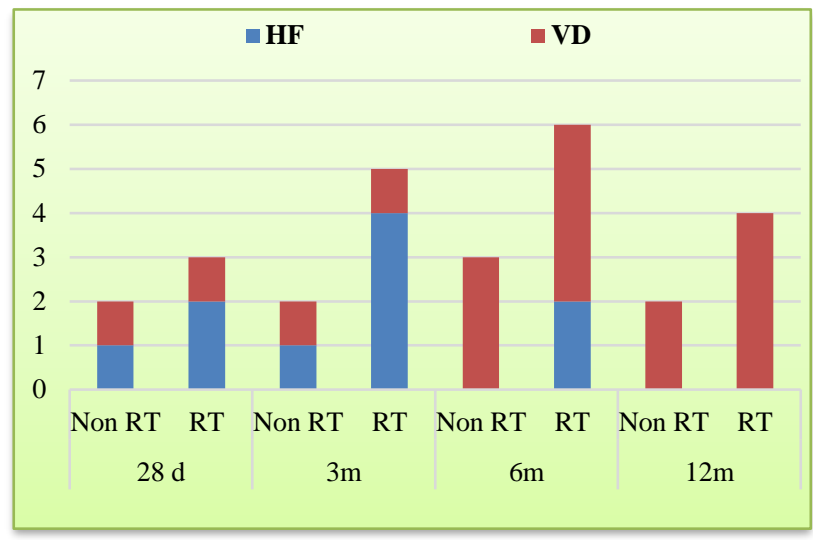

Symptomatology: Hot Flashes (HF) and Vaginal Dryness (VD).

\section{Figure 4: Symptoms related to ovarian failure}

On final analysis of the FSH levels a total of 7/19 (36.8\%) patients had FSH $>20 \mathrm{mIU} / \mathrm{ml}, 4$ from the RT group (50\%) 3 of whom had received EFRT and 3 from the no RT group (27\%). Thus, even in the RT group this procedure preserved ovarian function despite RT when standard dose was given with abdominal shielding (4/5 patients continued to have continued ovarian function). The rates of preservation of ovarian function were $63.2 \%$ overall and $72.7 \%$ in the No RT group.

\section{DISCUSSION}

Carcinoma cervix is treated by radical surgery in early stages with or without adjuvant RT. Removal of ovaries at surgery in young patients not only leads to symptoms of acute estrogen withdrawal but also deprives the patient the protection offered by estrogen against cardiovascular, cerebrovascular and osteoporotic complications and a healthy sexual life. OR at surgery was thought to be dangerous for fear of ovarian metastasis after some case reports of ovarian metastasis in transposed ovaries. However, studies have shown that ovarian metastasis is rare at less than $1 \%$ in squamous carcinomas and $1-2 \%$ in adenocarcinomas even in presence of pelvic nodal metastasis. ${ }^{10,11}$ This led to the practice of OR during surgery for Ca Cervix. However, many patients need adjuvant RT thus exposing the retained ovaries to the effect of radiation. The ovaries being exquisitely radiosensitive, doses as low as 10 Gray can be associated 
with a high risk of ovarian failure especially when the ovaries are directly in the field of radiation. ${ }^{12}$ Ovarian transposition has been found to be an effective means of preventing permanent damage to ovaries during radiotherapy.

Many techniques of ovarian transposition have been described depending on the final position of the ovary but irrespective of the surgical techniques, ovarian transposition is designed to place the ovaries outside the radiation field. The standard recommendation is that ovaries should be transposed $4 \mathrm{~cm}$ outside the radiation field which is generally achieved by placing it $1.5 \mathrm{~cm}$ above the iliac crest. ${ }^{13}$

Ovaries have been transposed medially behind the uterus when done for conditions like Hodgkin's lymphoma where the primary target are lymph nodes. However, in conditions like cervical malignancy where a lateral transposition outside the pelvis is required to keep the ovary away from the radiation field. The ovary may be placed intraperitoneally in the paracolic gutters or retroperitoneally on to the psoas muscle-both having its advantages and disadvantages.

Some studies have suggested that ovarian transposition cause premature ovarian failure after OR. This is due damage to the vasculature during the procedure and kinking of vessels especially when the ovary is tunneled retroperitoneally. Hysterectomy and or salpingectomy have also been quoted as reasons for ovarian failure after surgery also by compromising the vascularity. ${ }^{15,16}$ However no such effect was observed in this study. We used the surgical technique described by Belinson et al and the ovaries were fixed intraperitoneally near the lower pole of the kidneys. ${ }^{17}$ There were no difficulties experienced during the procedure and care was taken to avoid vascular injuries and kinking during the procedure. In our study the ovarian function continued in $63 \%$ which included $72.7 \%$ in non-RT group and $50 \%$ in RT group. Feeney et al. in a study of 28 women who underwent OR at $\mathrm{RH}$ reported a $40 \%$ continuation of ovarian function at 24 months while Chambers et al reported $71 \%$ ovarian function at 35 months in the absence of RT. ${ }^{18,19}$

Buekers et al observed that out of 24 patients who underwent Pelvic RT only $26 \%$ retained ovarian function at 1 year. ${ }^{20}$ Gubbala et al in their meta-analysis of 24 studies that included more than 800 patients also found that ovarian transposition was associated with significant preservation of ovarian function and negligible risk for metastases to the transposed ovaries. He found that $90 \%$ (95\% CI 92-99), of women had normal ovarian function when no RT was given and in the external beam radiotherapy (EBRT) group, the proportion of women with preserved ovarian function was $65 \%$ (95\% CI 56-74) and no metastases was reported in the transposed ovaries. ${ }^{4}$ Thus, the findings of the present prospective study are generally in agreement with the previous studies most of which are retrospective studies. The study also shows that even when eventually the ovaries failed, the transition was smoother, vasomotor symptoms milder and the need for HRT for symptom control was rarely required even in patients who received RT.

There has also been an apprehension regarding the practice of routine ovarian transposition in all patients undergoing $\mathrm{RH}$ for ca cervix rather than selective LOT only in patients with adequate ovarian reserve and likely to need adjuvant RT. This concern is due to two reasons. Firstly, some studies have shown that ovarian transposition reduces ovarian function appreciably possibly due to vascular compromise. Secondly, women older than 40 years are at higher risk for ovarian failure after any pelvic surgery due to ongoing age related decline of ovarian function thus making efforts of OR and LOT futile in these patients.

Selective LOT during OR for ca cervix may not be the right approach because, the assessment of the need for adjuvant RT prior to planned surgery is complex and fraught with many fallacies and so is assessment of ovarian function. Hence it would be unfair to deny the benefits of this procedure to patients who are willing and otherwise eligible for OR. In our study the mean age of patients was 41.2 years but all patients underwent assessment of ovarian function by FSH levels (mean FSH levels were 7.14) and only patients with actively functioning ovaries were considered for LOT. All patients also underwent Ca125 levels and TVS assessment of ovaries to screen for possibility of primary ovarian malignancy. Two patients in our study had high baseline FSH levels and were excluded from the study but there were no procedure related complications leading to ovarian failure.

The ideal candidate who would benefit maximum from LOT would be one who despite having an operable stage of Ca Cervix has a likelihood of receiving adjuvant RT, (Stage I B1 or more, a large volume disease, LVSI/PNI), has a low risk of ovarian metastasis and is below 40 years of age with actively functioning ovaries. Identifying this ideal candidate would be difficult especially in low resource settings. ${ }^{21}$ However, it would be unfair not to offer ovarian preservation with or without LOT in all eligible patients undergoing $\mathrm{RH}$ and desirous of conserving ovarian function.

\section{CONCLUSION}

LOT is an oncologically safe procedure and should be considered for all pre-menopausal patients undergoing RH for early stages of Ca cervix with low perceived risk of ovarian metastasis. LOT does not accelerate ovarian aging. Though chances of premature ovarian failure remain, which is higher after adjuvant $\mathrm{RT}$ and still higher when EFRT has to be given, the decline of ovarian function after LOT is gradual unlike in surgical menopause thus making the menopausal transition smooth. However, these patients need to be followed up 
for clinical and lab evidence of ovarian failure and any ovarian pathology.

Funding: No funding sources

Conflict of interest: None declared

Ethical approval: Not required

\section{REFERENCES}

1. McCall ML, Keaty EC, Thompson JD. Conservation of ovarian tissue in the treatment of carcinoma of the cervix with radical surgery. Am J Obstet Gynecol. 1958 ;75:590-605.

2. Webb GA. The role of ovarian conservation in the treatment of carcinoma of the cervix with radical surgery. Am J Obstet Gynecol. 1975;122:476-84.

3. Landoni F, Zanagnolo V, Lovato-Diaz L, Maneo A, Rossi R, Gadducci A et al. Ovarian metastases in early-stage cervical cancer (IA2-IIA): a multicenter retrospective study of 1965 patients (a Cooperative Task Force study). Int $\mathbf{J}$ Gynecol Cancer. 2007;17:623-8.

4. Gubbala K, Laios A, Gallos I, Pathiraja P, Haldar K, Ind $\mathrm{T}$. Outcomes of ovarian transposition in gynaecological cancers; a systematic review and meta-analysis. J Ovar Res. 2014;7:69.

5. Lushbaugh CC, Casarett GW. The effects of gonadal irradiation in clinical radiation therapy: a review. Cancer. 1976;37:1111-20.

6. Hodel K, Rich WM, Austin P, DiSaia PJ. The role of ovarian transposition in conservation of ovarian function in radical hysterectomy followed by pelvic radiation. Gynecol Oncol. 1982;13:195-202.

7. Anderson B, La Polla J, Turner D, Chapman G, Buller R. Ovarian transposition in cervical cancer. Gynecol Oncol. 1993;49:206-14.

8. Belinson JL, Doherty M, McDay JB. A new technique for ovarian transposition. Surg Gynecol Obstet. 1984;159:157-60.

9. Piver MS, Rutledge F, Smith JP. Five classes of extended hysterectomy for women with cervical cancer. Obstet Gynecol. 1974;44:265-72.

10. Yamamoto R, Okamoto K, Yukiharu T, Kaneuchi M, Negishi H, Sakuragi N et al. A study of risk factors for ovarian metastases in stage IB-IIIB cervical carcinoma and analysis of ovarian function after a transposition. Gynecol Oncol. 2001;82:312-6.

11. Zhao C, Wang JL, Wang SJ, Zhao LJ, Wei LH. Analysis of the risk factors for the recurrence of cervical cancer following ovarian transposition. Eur J Gynaecol Oncol. 2013;34:124-7.

12. Lo Presti A, Ruvolo G, Gancitano RA, Cittadini E. Ovarian function following radiation and chemotherapy for cancer. Eur J Obstet Gynecol Reprod Biol. 2004;113:S33-40.

13. Hwang JH, Yoo HJ, Park SH, Lim MC, Seo SS, Kang $S$ et al. Association between the location of transposed ovary and ovarian function in patients with uterine cervical cancer treated with (postoperative or primary) pelvic radiotherapy. Fertil Steril. 2012;97:1387-93.

14. Ellsworth LR, Allen HH, Nisker JA. Ovarian function after radical hysterectomy for stage IB carcinoma of the cervix. Am J Obstet Gynecol. 1983;145:185-8.

15. Plockinger B, Kolbl H. Development of ovarian pathology after hysterectomy without oophorectomy. J Am Coll Surg. 1994;178:581-85.

16. Menon RK, Okonofua FE, Agnew JE, Thomas M, Bell J, O'Brien PMS, Dandona P. Endocrine and metabolic effects of simple hysterectomy. Int $\mathbf{J}$ Obstet Gynecol. 1987;25:459-63.

17. Belinson JL, Doherty M, McDay JB. A new technique for ovarian transposition. Surg Gynecol Obstet. 1984;159:157-60.

18. Feeney DD, Moore DH, Look KY, Stehman FB, Sutton GP. The fate of the ovaries after radical hysterectomy and ovarian transposition. Gynecol Oncol. 1995;56:3-7.

19. Chambers SK, Chambers JT, Holm C, Peschel RE, Schwartz PE. Sequelae of lateral ovarian transposition in unirradiated cervical cancer patients. Gynecol Oncol. 1990;39:155-9.

20. Buekers TE, Anderson B, Sorosky JI, Buller RE. Ovarian Function after Surgical Treatment for Cervical Cancer. Gynecologic Oncol. 2001;80:85-8.

21. Yoon A, Lee YY, Park W, Huh SJ, Choi CH, Kim $\mathrm{TJ}$, et al. Correlation between location of transposed ovary and function in cervical cancer patients who underwent radical hysterectomy. Int $\mathbf{J}$ Gynecol Cancer. 2015;25(4):688-93.

Cite this article as: Jose T, Singh A, Sinha P, Bidari S. Ovarian preservation with lateral ovarian transposition in operable Ca cervix: experience at a tertiary care center. Int J Reprod Contracept Obstet Gynecol 2017;6:2337-41. 